\title{
REDES SOCIALES EN LA WEB, COMUNICACIÓN Y COMPORTAMIENTO SOCIAL
}

\section{Online social networks communication and social behavior}

Amaro La Rosa Pinedo*

\begin{abstract}
Resumen
El propósito del trabajo es presentar una visión del papel contemporáneo de las redes sociales en Internet y su impacto social. Se presenta un amplio análisis de las redes sociales, la comunicación y el comportamiento social basándose en la revisión de la investigación científica más reciente. Palabras clave: Internet, Redes sociales, Web 2.0, Comportamiento social.
\end{abstract}

\begin{abstract}
The main purpose is the presentation of an outlook of the contemporary role of online social networks and its social impact. The author presents a broad analysis of social networking, communication and social behavior based on a review of updated scientific research.
\end{abstract}

Key words: Internet, Social Networks, Web 2.0, Social behavior.

\footnotetext{
* Psicólogo Social y Comunicador. Docente de la Facultad de Traducción, Interpretación y Ciencias de la Comunicación, Universidad Femenina del Sagrado Corazón
} 
Lugar: Sala de conferencias de una universidad ubicada en Lima. Motivo: Conferencia. Expositor: Experto de una universidad europea quien entre otros temas se refiere a las redes sociales. Participantes: Docentes universitarios invitados de la especialidad de Comunicación. Conducta esperada: $100 \%$ del auditorio atento a la conferencia. Conducta observada: Tres docentes ubicados en la primera fila del auditorio tienen su Bluetooth encendido y pasan tiempo considerable revisando sus correos electrónicos y más que nada leyendo y escribiendo en su cuenta de Facebook. ¿Es tan urgente su necesidad de comunicación con la red social que no puede posponerse? Por cierto no se trata de ficción sino de un hecho real que observamos mientras preparábamos el presente artículo. Nos sirve como un caso para contextualizar nuestro tema. Los comentarios, conclusiones y tal vez los juicios de valor los dejamos para quien lea estas líneas

\section{BREVE INTRODUCCIÓN}

A través de la historia el ser humano se ha comunicado con sus semejantes de diversas maneras. Sus posibilidades se han ido incrementando y modificando en función de la evolución de la sociedad y del desarrollo de la tecnología, cuyo ritmo ha crecido aceleradamente en las últimas décadas del siglo XX y lo que va del XXI. En consonancia con ello, las redes sociales que integramos los actores sociales han variado igualmente en cuanto a las modalidades del intercambio comunicativo entre sus miembros. Con el surgimiento de la Web 2.0 ahora es posible actuar como emisores-receptores a nivel mundial, integrándose en redes que facilitan la producción de contenidos propios y el intercambio de mensajes de diverso orden entre los usuarios. En este breve trabajo intentamos aproximarnos a las variables psicosociales ligadas con el surgimiento y acelerado crecimiento de las redes sociales online.

\section{INTERNET Y COMUNICACIÓN MEDIADA POR COMPUTADORAS}

Nadie puede negar la importancia de Internet en la sociedad contemporánea, Un sinnúmero de investigaciones se han dedicado a explorar el terreno del comportamiento del ser humano en el ciberespacio y de las múltiples vinculaciones establecidas en este nuevo territorio virtual. Una simple entrada en los buscadores más conocidos nos da una aproximación a la importancia de esta temática

El ser humano interactúa permanentemente con su entorno social. Con el surgimiento de Internet aparecen nuevas formas de interacción. Se amplían así las posibilidades de ver el mundo, lo que entendido desde la difundida perspectiva de Mac Luhan supondría la extensión de los sentidos para ponernos en contacto con quienes están más allá de las fronteras de nuestras posibilidades perceptuales directas. En consonancia con ello las variables espacio-temporales se modifican sensiblemente.

A nuestro entender, y tal como lo explicamos en un trabajo previo (La Rosa, 2005) el impacto de Internet puede visualizarse en:

- Formas preestablecidas de comportamiento y comunicación (que ya existían antes de Internet).

- Nuevas modalidades de comportamiento y comunicación condicionadas por la propia naturaleza de Internet.

Habría que recoger el criterio de Liedelmain (1997) cuando toma en cuenta las influencias que pueden evidenciarse en las estructuras cognitivas por la propia utilización de un medio de esta naturaleza, que interviene como estímulo reforzador.

Otro de los desafíos viene por el lado del perceptor que en este caso es eminentemente activo y permanentemente se mantiene "en búsqueda de", ya sea que utilice el E mail, el Chat, las listas de interés, los motores de búsqueda y ciertamente las propias redes sociales. Se hace así realidad en el ciberespacio, la necesidad de diálogo que permanentemente requerimos satisfacer; aun cuando en este caso ello suponga la intermediación de un elemento propio del desarrollo tecnológico: la computadora, por lo cual se denomina a esta modalidad comunicación mediada por computadoras (CMC). Por cierto que en cuanto a este particular, no es lo mismo la comunicación sincrónica (aquella en que el mensaje se recibe luego de cierto periodo, como suele ocurrir con el correo electrónico) que la asincrónica (donde la interacción se hace efectiva casi de inmediato; en tiempo real. 
Noblia (1998) entiende que ya no es factible ubicar a la CMC dentro de los parámetros de la comunicación lingüística, pues se trata en este caso de modalidades de relación en los cuales no se comparte el mismo contexto social, proviniendo los interlocutores, en muchos casos de diferentes culturas, intercambiando datos que se basan en códigos que en su mayor parte se desconocen. Tanto más. A nuestro entender los propios paradigmas de la comunicación se modifican en tal dimensión que demandan un replanteamiento de criterios.
Para Millerand (1999) dada la situación en la cual la comunicación está mediatizada por la técnica, puede hablarse de una "tecnicización" de la misma relación de comunicación, lo que se pone en evidencia con la computadora y desde luego con Internet, donde a diferencia de los otros medios se demanda la participación permanente del perceptor. En cierto sentido coincide con este punto de vista Summerhill (1997) cuando establece una diferenciación entre las modalidades de comunicación a partir de las acciones del emisor y receptor. Por nuestra parte le agregaríamos una celda más a este cuadro en el cual consideraríamos a las redes sociales (Cuadro 1)

\section{CUADRO 1 \\ MODALIDADES DE COMUNICACIÓN \\ (Cada línea representa un proceso de comunicación)}

\begin{tabular}{lll}
\hline ACCION DEL EMISOR & CANAL DE COMUNICACIÓN & ACCION DEL RECEPTOR \\
\hline HABLA & $\begin{array}{l}\text { Onda de sonido } \\
\text { Cinta magnética }\end{array}$ & ESCUCHA \\
ESCRIBE & $\begin{array}{l}\text { Papel y tinta (carta) } \\
\text { Publicación formal (libro o revista) } \\
\text { Jeroglífico (u otro lenguaje iconográfico) }\end{array}$ & LEE \\
& Pared de caverna & VE/VISUALIZA \\
PINTA & Lienzo & \\
& Piedra (mármol o granito) & VE/VISUALIZA \\
ESCULTURA & Breve (Canto Gregoriano) & LEE \\
COMPOSICIÓN MUSICAL & Convencional \\
(Escritura) & Notación Musical \\
& Convencional (Occidental) & \\
& Instrumento Musical \\
& Interface Digital(MIDI) & \\
& Patch & \\
Onda de sonido & ESCUCHA \\
MUSICAL (Hablada) & Cinta magnética & \\
PROGRAMACIÓN DE & Mecanismo de computación & EJECUCIÓN DE PROGRAMA \\
COMPUTADORA & Red de cómputo (Gopher, Web, etc) & RECEPCIONA Y PRODUCE \\
REDES SOCIALES ONLINE & Internet (Recursos digitales múltiples) & \\
& & INFORMACIÓN \\
& &
\end{tabular}

Fuente: Summerhill (1997). Modificado por ALR 
Para Fernández - Maldonado (2004) en el Perú se está produciendo un notable impacto en los patrones de uso del tiempo libre así como el desarrollo de una cultura local en materia de Internet, lo cual se observa especialmente entre los jóvenes. Por nuestra parte hemos observado modificaciones en el universo discursivo, pues el repertorio lingüístico contemporáneo ha incorporado una serie de expresiones que son parte del argot de sistemas y de Internet. En el habla cotidiana de las ciudades peruanas la expresión "Ir a Internet" se emplea como sinónimo de acercarse a una cabina pública, y la propia palabra Internet puede entenderse con tres significados:

- El medio de comunicación.

- El espacio físico donde opera una cabina pública.

- La situación de estar interconectados con alguien a través de la CMC

\section{LA IMPORTANCIA DE LAS REDES SOCIALES EN EL MUNDO CONTEMPORÁNEO}

Hoy en día se habla constantemente de redes sociales. Pero cabe preguntarnos $¿$ sabemos lo que implica en términos generales una red social? Desde el punto de vista de Butts (2008) consiste en un conjunto de entidades (personas, grupos) que tienen alguna relación entre sí. Cada individuo es naturalmente diferente del otro lo que permite identificarlo y su número es finito.

La teoría de las redes sociales estima que en la vida diaria formamos diversas redes sociales, de características distintas, con diferente grado de importancia para cada uno de sus integrantes.

Por cierto las redes sociales como modalidades de intercambio social se han formado desde las primeras épocas de existencia del ser humano. Tanto más, Fowler, Dawes \& Christakis (2009) realizaron un estudio según el cual pudieron establecer que las redes sociales individuales tienen una base parcialmente genética, exhibiendo patrones sistemáticos similares en diversos contextos humanos. Anotan que por lo menos tres de los rasgos que se observan de naturaleza hereditaria.
No obstante, con el advenimiento de la web 2.0 se desarrollan cada día más las redes sociales online. Así, las posibilidades de comunicación se han incrementado notoriamente. Cada día millones de personas en el mundo entero comienzan su jornada encendiendo su computadora apenas se levantan, revisando los últimos datos de sus contactos en Facebook, enviando datos sobre sus acciones en Twitter, colgando sus videos en Youtube o sus fotografías en Flickr. Y ahora contando con los teléfonos móviles inteligentes, se mantienen en contacto en tiempo real realizando estas mismas operaciones desde cualquier lugar donde se encuentren

Si quisiéramos relacionarnos con cualquier otro ser humano en la tierra teóricamente solamente nos bastarían seis pasos para llegar a su encuentro. Esta es la afirmación que deviene del estudio que erróneamente se atribuye a Milgram y que ha tendido en denominarse la Teoría de los seis grados de separación (Gráfico 1). Una de las leyendas urbanas, que también se tejen en el mundo de la ciencia, afirma que sus fundamentos aparecen en Milgram (1969) y que se desarrollan en Travers y Milgram (1969), Si queremos investigar, una revisión acuciosa de la evidencia de las fuentes nos aproximará a la verdad.

\section{GRÁFICO 1}

\section{LA TEORÍA DE LOS SEIS GRADOS}

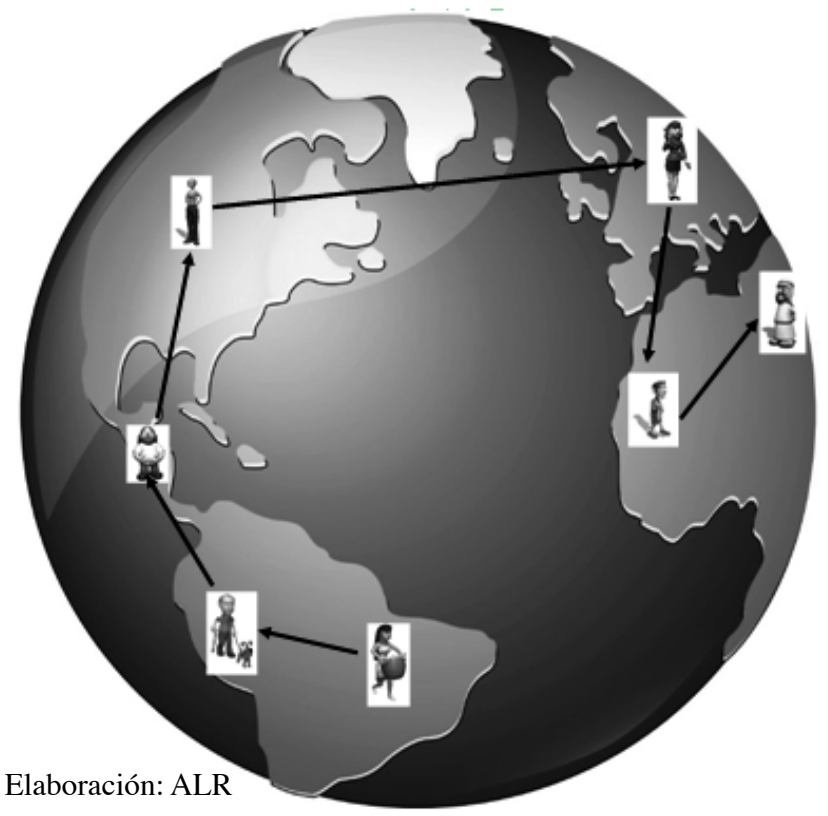


Gewrloff (2006) afirma que vivimos en una sociedad en red, lo cual implica que las redes constituyen la característica estructural dominante de la presente era de la información. Villanueva (2010) coincide con esta perspectiva, cuando expresa que sin una red no tenemos dónde vivir y que para acceder a la vida digital, es una necesidad primordial el acceso a las redes que le dan existencia.

Las nuevas tecnologías atraen en especial a la población joven que tal como se conoce, constituye la mayoría en nuestro país. Es evidente que los jóvenes ven a Internet como una gran posibilidad para ensanchar sus horizontes y ponerse en contacto con personas de diversos lugares del mundo; y es que son los denominados nativos digitales que han nacido en un mundo donde Internet es una realidad omnipresente. No obstante, Madden (2010) informa que el uso de redes sociales en Internet está creciendo aceleradamente entre los adultos de mediana edad, quienes suelen integrar el grupo de los denominados inmigrantes digitales (El gráfico 2 muestra una comparación entre ellos). El informe reveló que entre adultos de 50 a 64 años, el uso de redes sociales subió un 88 por ciento en el período comprendido entre abril de 2009 y mayo de 2010, y totalizó un 47 por ciento de la población en estudio. Para aquellos

\section{Gráfico 2 \\ COMPARACIÓN ENTRE NATIVOS E INMIGRANTES DIGITALES}

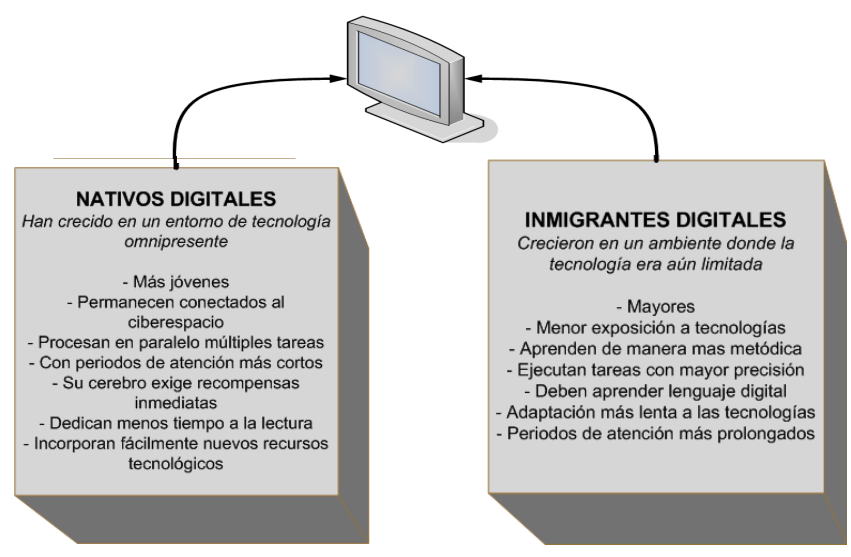

Fuentes: Prensky (2001a y 2001b) Small y Vorgan (2009). Elaboración: ALR de 65 años y más, el uso de las redes sociales exhibió un aumento de 100 por ciento, desde un 13 por ciento inicial a un 26 por ciento en mayo. En contraste, el uso de las redes sociales en Internet entre la población de entre 18 y 29 años creció sólo un 13 por ciento, y alcanzó a un 86 por ciento de este grupo etario.

A decir de Veber (2010), las redes sociales son entornos en línea que conectan a las personas de acuerdo a sus intereses y que se construye entre las empresas y los usuarios individuales. Configuran nuevas herramientas estratégicas que abren oportunidades para formar e influir en la opinión pública, a cada minuto.

La web 2.0 supone comunidad, colaboración y comunicación. La arquitectura de red y las tecnologías de la web 2.0 ofrecen algunos rasgos básicos que a decir de Muruguesan, (2010) son los siguientes:

- Facilitan un flexible diseño web, reuso creativo y rápida actualización.

- Proporcionan una interfase de usuario rica y responsiva.

- Soportan la colaboración y la asistencia en la recolección de inteligencia colectiva.

- Facilitan la creación de contenido colaborativo y la modificación por parte de los usuarios.

- Establecen redes sociales de personas que tienen intereses comunes.

- Posibilitan la creación de nuevas aplicaciones atractivas reusando, combinando y/o fusionando diferentes aplicaciones en la web o combinando data e información de diferentes fuentes.

Por nuestra parte, mencionamos algunos aspectos de la web 2.0 que la hacen más asequible y facilitan la creación de contenidos por parte de los usuarios:

- Simplicidad en el uso pues quien es usuario de una red social se encuentra con un sofware muy amigable que le permite manejarse con mucha sencillez ${ }^{1}$.

- No requiere aprenderse el HTML ni ningún otro lenguaje complejo, tal como el de las primeras generaciones de computadoras.

1 Desde luego, esto funciona para quien tiene las competencias necesarias para el uso de computadoras y del acceso a Internet. 
- Placer de utilización: El usuario puede pasarse hora tras hora conectado con el mundo de la red social que le interesa, dada la naturaleza de los contenidos que encuentra, que se incrementan constantemente y los que puede ingresar en su cuenta que lo hacen mantenerse inmerso en el sistema.

- Separación de forma y contenido: Se acercan mucho ambos pues es el usuario quien aporta el volumen más importante de contenido, por cierto que sobre la base de un software preestablecido, de uso gratuito el cual ni siquiera requiere instalarse y que más aún recibe los aportes de sus usuarios quienes como verdade- ros desarrolladores producen utilitarios y aplicaciones que amplían los horizontes de la red social. Tal es el caso de Facebook que ha incrementado el interés de los usuarios en tanto ha incorporado un sinnúmero de recursos útiles, interesantes o motivadores desarrollados por sus usuarios.

En la web 2.0 y tal como lo intentamos ilustrarlo (Gráfico 2) los usuarios producen sus propios contenidos y se convierten en consumidores -.productores o producidores, para usar un término acuñado por el futurólogo Alvin Toffler cuatro décadas atrás (Toffler, 1973)

\section{GRÁFICO 3 WB 2.0 Y PROSUMIDORES}

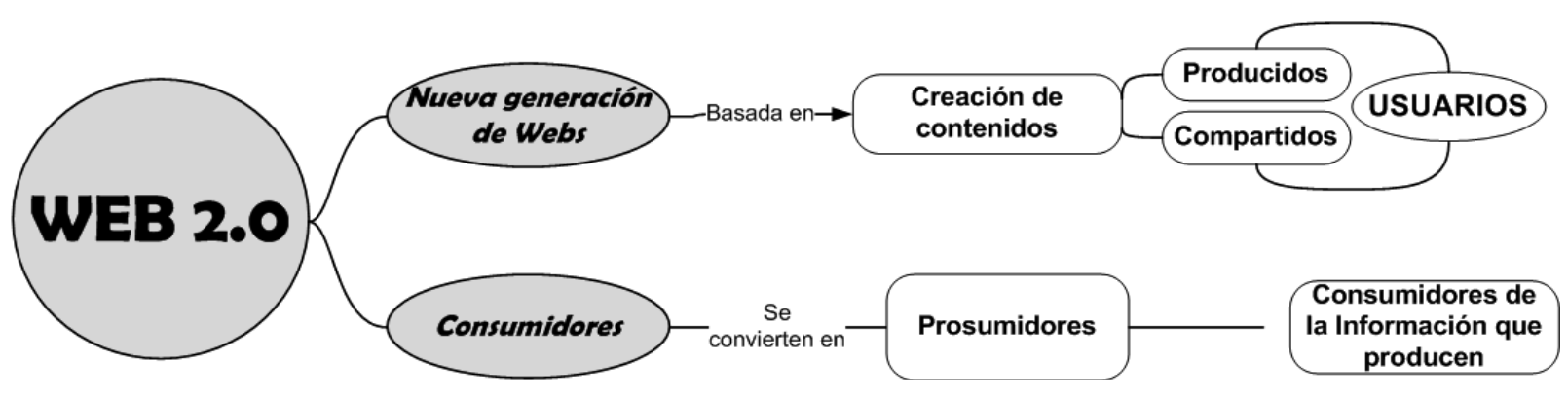

Según las mediciones de Alexa (2010) entre los 50 sitios más visitados en Internet a nivel global figuran varias redes sociales:
1. Facebook
2. Youtube
3. Blogger
4. Twitter
5. QQ.com (red social china)
6. Word Press.com
7. Linkedin
8. Myspace
9. Flickr
10. V Kontakte (red social rusa)

Cunha (2006) define a las redes como un conjunto integrado por dos factores: actores y sus conexiones, entendiendo a éstas como provenientes de procesos de comunicación. Realiza una clasificación de las comunidades online sobre la base de los fotologs ${ }^{2}$

1. Emergentes: Interacción social mutua, generando un sentimiento de pertenencia al grupo, basado en intercambios dialógicos, incluyen un número limitado de integrantes y tienden a ser menos estables. Serían más cohesionados basados en lazos fuertes con reciprocidad y confianza

2. De asociación: Interacción social relativa, se construye una pertenencia asociativa, basada en intercambios más formales. Constituyen grupos grandes de

2 Páginas personales que permiten compartir fotografías 
mayor estabilidad pues no dependen de los intercambios para mantenerse, menos cohesionados con lazos menos intensos

3. Híbridas de nivel intermedio. pueden implicar una estructura de varios clusters más conectados que las asociativas, pero menos que las emergentes. Pueden tener fases que lo acercan más a uno que a otro de estos tipos.

\section{REDES SOCIALES, COMUNICACIÓN Y COM- PORTAMIENTO SOCIAL}

No requiere constatación empírica la presunción de que necesitamos ser escuchados por los demás, lo que forma parte del gregarismo propio del ser humano. Las características de la sociedad contemporánea condicionan múltiples contactos superficiales, observándose los rasgos anómicos de los que hablara Merton, los cuales involucran la disminución de los contactos realmente humanos, frente a frente. De allí que exista una necesidad insatisfecha de comunicación, la cual en algunos casos es reemplazada por las formas diversas de intercambio que ahora hace posible Internet. En nuestro trabajo cotidiano observamos que estas modalidades de comunicación son tanto más necesarias cuanto más solitario es el individuo. Sus reales déficits de comunicación con su entorno social son superados en cierta medida gracias a la magia de la comunicación mediada por computadoras, en la cual por más que el individuo tenga la ilusión de comunicarse directamente con la otra persona, lo cierto es que la intermediación no deja de hacerse efectiva, pues la máquina no solamente está presente siempre sino que es un recurso indispensable para que se establezca y mantenga el vínculo.

Obviamente todos los usuarios no poseen las mismas características. Sobre el particular Ofcom (2008) realiza una clasificación de los usuarios de las redes sociales considerando los siguientes tipos:

- Alfa Socializadores: Constituyen una minoría que usa los sitios sociales en intensos y cortos periodos de tiempo para flirtear, conocer gente nueva o entretenerse (Hombres, por debajo de los 25 años).

- Buscadores de Atención: Algunas personas quienes buscan la atención y los comentarios de los demás, posteando fotos y personalizando sus perfiles (Mujeres y adolescentes hasta los 35 años).

- Seguidores: Personas que en gran cantidad se unen a sitios sociales para estar al día de las actividades de sus contactos en la vida real (Mujeres y hombres, de todas las edades).

- Fieles: Personas que en gran número usan las redes sociales para recuperar amistades del pasado. (Hombres, mujeres, mayores de 20).

- Funcionales: Algunas personas quienes tienden a usar los sitios de redes sociales con un único objetivo, puntual (Hombres, mayores de 20).

Rainie (2005) sugiere que la auto expresión, opuesta a la interacción, es la razón principal de los individuos para decidir convertirse en bloguero ${ }^{3}$, Por su parte Fullwood, Sheehan \& Nicholls (2009) en el marco de un estudio sobre los blogs de MySpace encuentran tres motivaciones fundamentales para bloguear: Autoexpresión, establecimiento de contactos en red4 y gestión de la identidad.

Lameiro y Sánchez (1998) realizaron un estudio cualitativo sobre los vínculos de Internet entre usuarios, tomando como muestra cibernautas de diversas culturas (entre otros, de Argentina, Perú, España, Italia e Israel). Encontraron que la motivación fundamental es el establecimiento de comunicación con otras personas, en el entendido que ello involucra una notoria y sustancial dimensión afectiva.

Las redes sociales no necesariamente movilizan a las personas de su mundo real de interacción. Más bien se emplean para apoyar las relaciones y mantenerse en contacto a pesar de los cambios en las distancias establecidas entre las personas (Lewis, 2007). Sobre este particular Baym (2009) estima que las relaciones con otras personas son de manera creciente multimodales y cuanto más cercanas son las relaciones entre las per-

\footnotetext{
3 Bloguero: Usuario de blog

4 Networking en el original. Traducción: ALR
} 
sonas más recursos de comunicación las unen. Así, una pareja de enamorados que estudian juntos en una universidad y se comunican prácticamente durante todo el día, empiezan a intercambiar mensajes de texto y/o a conversar a través de sus celulares apenas se separan, se conectan apenas llegan a sus respectivas casas usando la CMC y no sería raro que sigan interactuando nuevamente durante horas empleando Skype, Messenger o ICQ, sintiéndose "más cercanos" porque se ven a través de sus webcams.

Cabría preguntarnos aquí ¿qué pasa con aquellas personas que se aíslan de los suyos y se dedican más que nada a la comunicación electrónica con otros pares lejanos? En este caso volvemos a ingresar en un terreno sobre el cual se ha caminado insistentemente, dando muchas veces pasos en falso y sobre el cual se han escrito centenares de versiones antojadizas, de esas que abundan en la red de redes. En este caso, no es la tecnología la culpable del aislamiento del individuo, pues éste ya se comportaba de la misma manera antes de que la tuviera en sus manos.

Milani, Osualdella, \& Di Blasio (2009) afirman que los adolescentes con relaciones interpersonales limitadas y una predisposición por estrategias de evitamiento en la solución de problemas, tienen un mayor riesgo de adicción a Internet. En este caso usan Internet con propósitos socializadores, que paradójicamente no aplican en sus relaciones interpersonales cotidianas.

La investigación realizada por Tahiro_lu, Çelik, Fettaho_lu, Yıldırım, Toros, Avcı, Özatalay.\& Uzel (2010) en una muestra de 884 adolescentes de 12 a 18 años, encuentra una relación estrecha entre adicción a Internet y desórdenes psicopatológicos entre los cuales destaca el déficit de atención con hiperactividad. El estudio comprueba evidencias previas sobre el particular. Así,

Yoo, Cho, Ha, Yune, Kim. Hwang, Chung, Sung. y Lyoo (2004) describen asociaciones significativas entre niveles de síntomas de déficit de atención con hiperactividad y severidad de adicción a Internet en niños.
El estudio de Pelling y White (2009) revela que un alto nivel de tendencias adictivas hacia el uso de mensajes escritos en redes sociales se relaciona de manera significativa con necesidades de pertenencia e identidad de sí mismo. Basándose en la teoría de usos y gratificaciones ${ }^{5}$, Park, Kerk. \& Valenzuela (2009) encuentran que los estudiantes universitarios se unen a grupos de Facebook por su necesidad de socializarse con los amigos y de búsqueda de estatus.

El nivel de popularidad que experimentamos en la vida diaria, esto es la evidencia de ser percibido como sujeto valioso por sus amigos y por la sociedad, quien sabe pueda entenderse en vinculación con la importancia que la persona tiene para su comunidad y para sus pares más cercanos. En cambio en el mundo de las redes sociales online, resulta más valioso, de mayor prestigio, quien cuenta con el mayor número de contactos agregados a su cuenta. La investigación de Madden y Smirh (2010) demuestra que más de la mitad de los usuarios adultos de Internet (57\%) emplearon motores de búsqueda para buscar información sobre sí mismos y para conocer cual era la clase de información disponible.

Del nivel de popularidad que otorgan las redes sociales dan fe los políticos quienes las utilizan para difundir su propaganda. Sobre el particular, uno de los factores que facilitó el triunfo electoral de Barack Obama en Estados Unidos fue precisamente el uso apropiado de las redes (Baella, 2008)

Los criterios de Goffman sustentados hace varias décadas resultan ahora plenamente vigentes para explicar las estrategias de auto-presentación de las personas en Facebook. Ello ocurre según Zhargouni (2007) porque los mecanismos de auto presentación son parte importante de nuestra vida diaria. Y están tan fuertemente arraigados en el autoconcepto que devienen en patrones aplicables a diversas puestas en situación.

Las nuevas modalidades de auto-presentación en las redes sociales son estudiadas por Mehdizadeh (2010) quien luego de estudiar a un grupo de 100 usuarios de Facebook revela que los individuos con altos puntajes

5 La teoría de usos y gratificaciones establece que existen una serie de aspectos sociales que están en la base de necesidades psicológicas que predisponen a los individuos a utilizar aquellos contenidos de los medios de comunicación que les resultan gratificantes. 
de narcisismo y bajos en autoestima registran una constante actividad en la red social donde se esfuerzan por mostrar contenido promocional. De modo similar, Buffardi, \& Campbell (2008) analizando auto reportes narcisistas de usuarios de Facebook encontraron que registraban mayor cantidad de interacción online, fotografías principales de auto promoción y fotografías caracterizadas por su atractivo.

Silva-Peña, Marchant, Gonzales y Novoa (2006) afirman que los jóvenes viven cotidianamente experiencias de comunicación online muchas veces anónimas las cuales validan como modalidades de comunicación entre pares, originando redes de contacto en las cuales interactúan con un lenguaje propio, cuya evidencia hemos podido observar por nuestra parte, de manera empírica, en estudiantes universitarios de la capital peruana.

Desde la perspectiva de Costa (2005), sabemos a qué redes pertenecemos pero no puede conocerse fácilmente a cuáles pertenecen otros, lo cual ocurre tanto en las relaciones online como en las offline. Un aspecto esencial para la consolidación de las redes es la confianza mutua entre las persona, que está relacionada directamente con la posibilidad de percibir al otro e incluirlo en su universo de referencia. Con las redes sociales online se encuentran zonas de proximidad entre personas sin limitaciones de tiempo y espacio, aún cuando esas proximidades, insistimos, estén siempre intermediadas por la tecnología.

Por cierto que no en todos los aspectos de la comunicación hay un criterio homogéneo. Lewis (2007) encuestó a 1,222 estudiantes de dos grandes universidades públicas de Estados Unidos. Comparando los datos de hace cinco años con los de ahora, encontró que los jóvenes adultos esperan menos de las noticias e información procedente de las redes sociales y más de los medios tradicionales, especialmente de los diarios.

El Perú es heredero de la ancestral cultura andina, uno de cuyos principales rasgos es el intercambio de la solidaridad. Ella sobrevive en la era del ciberespacio, en contraposición con las agoreras profecías de los luditas ${ }^{6}$ del proceso de globalización, quienes suponen que las culturas locales están condenadas a su extinción. En lo últimos años, por intermedio de la red se han puesto en marcha innumerables cadenas de solidaridad entre las cuales podemos mencionar algunas destinadas a salvar la vida de personas que requerían de tratamientos médicos que no estaban a su alcance o para colaborar con los afectados por el fenómeno del Niño o el friaje (ola de frío) en la zona altiplánica del sur peruano.

Una investigación realizada en Cusco, Iquitos y Chiclayo, entre escolares de dos grupos de edad (1112 y 15-16 años) muestra que se vienen produciendo una serie de cambios importantes en el acceso a las tecnologías de la información y la comunicación, a un ritmo bastante acelerado. Internet resulta un espacio nuevo de encuentro con los amigos, de conversación y de comunicación en general que les permite interactuar con otros, y decirse cosas que tal vez no se atreven en una interacción cara a cara. Internet es para los más jóvenes de esas ciudades una tecnología de relación, una cultura de comunidad donde encuentran satisfacción y comparten estados de ánimo a la distancia. Quiroz (2008). Encontramos un visión similar en Fullwood, Sheehan \& Nicholls (2009) quienes estiman que el blogueo contribuye al bienestar emocional, especialmente de quienes tienen dificultades para expresarse ante los demás en una interacción cara a cara: especialmente si se trata de asuntos demasiado personales. Ciertamente resulta paradójico confiar en alguien a quien se conoce a través de Internet y quien tal vez esté muy lejos, físicamente hablando.

De acuerdo a Tuten (2008) la construcción de la identidad en las redes sociales se basa en los perfiles, cuyo primer rasgo es el gráfico (o la fotografía) que el usuario emplea para representarse incluyendo asimismo todos los otros datos que la persona completa cuando se registra y que la red solicita. Pero la identidad también incluye a sus amigos (en la red), los posts colocados por el usuario, los comentarios de sus amigos y las afiliaciones que se registran.

Es habitual que un usuario pueda cambiar esta "identificación personal" cuantas veces lo desee. So-

6 Obreros que a comienzos de la Revolución Industrial destruían las máquinas a vapor de las fábricas inglesas. 
bre el particular, la investigación de Zywica y Danowski (2008) demuestra que a menudo, los usuarios populares suelen cambiar los gráficos de su perfil con mayor frecuencia.

Hay en suma, diversidad de evidencias científicas que analizan el mundo de las redes sociales en su vinculación con el comportamiento social. Nos detenemos por ahora en este análisis que congrega cada día a un mayor número de especialistas. La nuestra es una tarea inconclusa que esperamos ir completando más adelante, pero que como ocurre con el estudio de la comunicación experimenta variaciones constantes que involucran nuevas exigencias para el trabajo de investigación.

\section{REFERENCIAS}

Alexa The Web information company (2010) Top Sites. The top 500 sites on the web. Recuperado el 10 de setiembre de 2010 de http://www.alexa.com/topsites

Baella, A. (2008) Yo quiero ser Presidente. Lima: Baella Consulting

Baym, N. (2009) A call for grounding in the face of blurred boundaries. Journal of Computer-Mediated Communication, 14 (3) 720-723

Buffardi, L. \& Campbell, W. (2008) Narcissism and social networking Web sites. Personaliry and Social Psychology Bulletin, 34 (10), 1303-1314

Butts, C. (2008) Social network analysis: A methodological introduction. Asian Journal of Social Psychology, 11, 13-41

Costa, R. (2005) Por um novo conceito de comunidade: redes sociais, comunidades pessoais, inteligência coletiva. Interface-Comunicação, Saúde, Educação., 9, (17), mar/ago. 235-248

Cunha, R. da (2006) Comunidades em redes sociais na Internet. Tese Doutorado em Comunicação, Universidade Federal do Rio Grande do Sul

Fernández-Maldonado, A. (2004) Los cibercafés: ¿un arma útil contra la brecha digital? e.Learning Europe of the European Union. June. Recuperado el 10 de setiembres de 2010 de: http:// w w w. e le a r n i g e u ropa.info/ doc.php?lng=8\&id=5077\&doclng=1\&p3=1

Fowler, J., Dawes, C. \& Christakis, N. (2009) Model of genetic variation in human social networks. PNAS, 106(6), February, 1720-1724

Lameiro, Máximo y Sánchez, Roberto (1998). Vínculos e Internet. Revista Campo Grupal No 1, Bs. As. Julio - Agosto . Disponible en http://www.geocities.com/ SoHo/Museum/ 9653/vinculos.htm

Fullwood, C., Sheehan, N. \& Nicholls, W. (2009) Blog Function Revisited: AContent Analysis of MySpace. Blogs CyberPsychology \& Behavior, December, 12(6), 685-689.

La Rosa, A. (2005) Interacción social en el ciberespacio. En: Islas, O., Editor, Internet y la Sociedad de la Información. Tomo 1, pp.207-224. Quito: CIESPAL.

Lewis, S. (2007) Next-generation news consumers: An exploratory study of young adults and their future with news and newspapers. Paper presented at the Annual Meeting of the Association for Education in Journalism and Mass Communication, The Renaissance, Washington, DC, August

Liedlmair, K. (1999) From the Philosophy of Technology to a theory of media, Society for Philosophy \& Technology, 4(3). Disponible en :http:// scholar.lib.vt.edu/ejournals/SPT/v4_n3html/ LIEDLMAI.html

Madden, M. \& Smith, A. (2010) Reputation Management and Social Media: How people monitor their identity and search for others online. Washington, D.C : Pew Internet \& American Life Project. Recuperado el 26 de mayo de 2010 de: http://pewinternet.org/ /media/ / F i l e s / R e p o r t s / 20010 / PIP_Reputation_Management_with_topline.pdf

Madden, M. (2010) Older Adults and Social Media: Social networking use among those ages 50 and older nearly doubled over the past year. Washington, D.C: Pew Internet \& American Life Project, August 27. Recuperado el 10 de setiembre de 2010 de: http:// www.pewinternet.org/ /media//Files/Reports/2010/

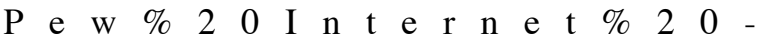
$\%$ 20Older\%20Adults\%20and\%20Social\%20Media.pdf

Mehdizadeh S. (2010) Self-presentation 2.0: narcissism and self-esteem on Facebook Cyberpsychology, Behavior and Social Networking, 13(4), 357-364

Milani, L. Osualdella, D. \& Di Blasio, P. (2009) Quality of Interpersonal Relationships and Problematic Internet Use in Adolescence. CyberPsychology \& Behavior, 12(6): 681-684.

Milgram, S. (1967) The Small World Problem, Psychology Today, 2, 60-67 
Millerand, F. (1999) Usages des NTIC:les approches de la diffusion, de l'innovation et de l'appropiation (2e partie) COMMposite v 99.1. Disponible: http:// commposite/uqam/ca/99.1/articles/ntic_2.htm

Muruguesan, S. (2010) Web X.0: A Road Map. In Muruguesan, S. Web 2.0, 3.0 and X.0: Technologies, Business and social applications. Hershey, Pennsylvania: International Science Reference, pp 111

Noblia, M. V. (1998), The Computer_Mediated Communication, a Way of Understanding The Language, International Conference, 25-26 March, Bristol, UK. Disponible http:// www.sosig.ac.uk(iriss(papers/paper22.htm

Ofcom (2008) Social Networking: A quantitative and qualitative research report into attitudes, behaviours and use. London: Office of Communication. Disponible http://stakeholders.ofcom.org.uk/binaries/ research/media-literacy/report 1.pdf

Park, N., Kerk F. \& Valenzuela, S. (2009) Being Immersed in Social Networking Environment: Facebook Groups, Uses and Gratifications, and Social Outcomes. CyberPsychology \& Behavior, 12(6): 729-733.

Pelling, E. \& White, K. (2009) The Theory of Planned Behavior Applied to Young People's Use of Social Networking Web Sites. CyberPsychology \& Behavior, 12(6), 755-759.

Prensky, M. (2001a) Digital Natives, Digital Immigrants Part 1. On the Horizon, 9(5), 1-6

Prensky, M. (2001b) Digital Natives, Digital Immigrants Part 2 Do They Really Think differently? On the Horizon, 9(6), 1-6

Quiroz, M. T. (2005). Jóvenes e Internet. Entre el pensar y el sentir. Lima: Fondo Editorial de la Universidad de Lima,

Quiroz, M. T. (2008). La edad de la pantalla. Tecnologías interactivas y jóvenes peruanos. Lima: Fondo Editorial de la Universidad de Lima.

Rainie L. (2005, May 2) New data on blogs and blogging. Pew Internet \& American Life Project. Recuperado el 5 de noviembre de 2010 de: www.pewinternet.org $=$ Commentary $=2005=$ May $=$ Newdataon-blogs-and-blogging.aspx

Fecha de recepción: 10 de setiembre, 2010

Fecha de aceptación: 25 de setiembre, 2010
Silva-Peña, I., Borrero, A., Marchant, P., González, G. y Novoa, D. (2006) Percepciones de jovenes acerca del uso de las tecnologias de informacion en el ambito escolar. Ultima década, 14 (24), 37-60. Disponible: http://www.scielo.cl/scielo.php? pid=S0718$22362006000100003 \&$ script=sci_arttext

Small, G. y Vorgan, G. (2009) El cerebro digital. Barcelona: Urano

Summerhill, C. (1997) Computer-Mediated Communication as Publication: Considering the World Wide Web in the Broader Sociological Context of Communication. Documento on line. Disponible: http://staff.cni.org/-craig/castalks/cmc.html

Tahiro_lu, A., Çelik, G., Fettaho_lu, C., Yıldırım, V., Toros, F., Avc1, A., Özatalay, E. \& Uzel, M. (2010) Psikiyatrik Bozuklu_u Olan ve Olmayan Ergenlerde Problemli_nternet Kullanımı Nöropsikiyatri Arflivi, 47, 241-246

Toffler, A. (1973) El shock del futuro Barcelona: Plaza \& Janés.

Travers, J., \& Milgram, S. (1969), An Experimental Study of the Small World Problem. Sociometry 32(4), December, 425-443.

Tuten, T. (2008) Advertising 2.0: Social media marketing in a web 2.0 world. Westport, Connecticut: Praeger Publishers.

Villanueva, E. (2010) Vida digital: La tecnología en el centro de lo cotidiano. Lima: Fondo Editorial Pontificia Universidad Católica del Perú.

Yoo, H., Cho, S., Ha, J., Yune, S., Kim. S., Hwang, J., Chung, A., Sung, Y. y Lyoo, I. (2004) Psychiatric and Clinical Neurosciences, 58 (5), 487- 494.

Zhargouni, S. (2007) A Study of Self-Presentation in Light of Facebook. Oslo: Institute of Psychology, University of Oslo, Autumn.

Zywica J, y Danowski, J. (2008) The face of Facebookers: Investigating Social Enhancement and Social, Compensation Hypotheses; Predicting Facebook and Offline Popularity from Sociability and Self-Esteem, and Mapping the Meanings of Popularity with Semantic Networks. Journal of Computer-Mediated Communication, !4, 1-34 